УДК 532.2, 541.14, 547.97

\title{
Laser-induced Interaction of Multilevel Quantum Dots
}

\author{
Andrey A. Glushkov* \\ Evgenia A. Slyusareva ${ }^{\dagger}$ \\ Aleksander S. Aleksandrovsky ${ }^{\ddagger}$ \\ Aleksey S. Tsipotan ${ }^{\S}$ \\ Vitaliy V.Slabko \\ Institute of Engineering Physics and Radio Electronics \\ Siberian Federal University \\ Svobodny, 79, Krasnoyarsk, 660041
}

Russia

Received 13.06.2016, received in revised form 14.09.2016, accepted 20.11.2016

A theoretical model is developed for describing the laser-induced interaction of a pair of multilevel semiconductor quantum dots. Spectral dependencies of interaction energy are calculated in dipole-dipole approximation for a pair of CdSe quantum dots in a quasi-resonant laser field, for cases of two identical quantum dots and those with differing transition wavelengths. Interaction energy of pair of multilevel quantum dots in the long-wavelength potential well is much higher than that of pair of two-level quantum dots. The increase of difference of resonance wavelengths of two interacting quantum dots leads to decrease of the potential well depth.

Keywords: laser-induced interaction, nanoparticles and nanustructures, Kramers-Kronig relation. DOI: $10.17516 / 1997-1397-2017-10-1-108-116$.

The studies of the methods of producing nanostructures with pre-defined characteristics is presently of keen interest. Its worthy to note reviews devoted to the problems of nanocrystals' formation from metal nanoparticles $[1,2]$, as well as from semiconducting and diectric materials [3-6]. The interest to the self-organization of nanoparticles into complex structures is common for these studies, the mechanisms of the former being actively studied $[7,8]$. Self-organization based on selective chemical interactions of molecules is studied in [9-11]. Prospective extension of this trend is the self-organization based on physical interactions, allowing to control both the structures' growth process and the resulting shape of them. Among physical interactions one can distinguish, e.g., electric, mechanical, convective and optical ones [1].

Recently a method of self-organization of nanoparticles into the structures with the predefined geometry in the field of resonant laser radiation was suggested [12,13]. It was shown that under corresponding choice of the wavelength and the polarization of external laser field with respect to the structure under formation the interaction energy of dipoles induced on the particles can exceed the thermal motion energy. The method allows to form structures with desired pre-defined geometry from several nanoparticles of different materials in the course of

\footnotetext{
*krasattache@gmail.com

$\dagger$ ESlyusareva@sfu-kras.ru

†aleksandrovsky@kirensky.ru

§cipotana@mail.ru

ๆvslabko49@mail.ru

(C) Siberian Federal University. All rights reserved
} 
multistage self-assembly process. In the following, using the mathematical modeling methods, the influence of the phase difference of induced dipole moments [14], and of the height of the barrier preventing from spontaneous aggregation [15] were studied.

Evidently, a pair of particles is the most suitable configuration both for experiment and modeling. The presence of aggregated pair of nanoparticles can be easily monitored via spectral methods. Spectral properties of a pair of metallic particles as the function of interparticle distance were studied in $[16,17]$. Formation of pairs via photochemical reactions was studied in [18], as well as in the conditions of spatially modulated laser irradiation [19,20].

Electromagnetic interaction of nanoparticles induced by laser irradiation was earlier considered in the single-resonance approximation [12-15] This approach is justified for metal particles with surface plasmon resonance [21]. Formation of pre-defined nanostructures of semiconducting quantum dots (QD) [22], and dielectric nanoparticles with resonant rare earth or transition metal dopants [23] is more prospective. Spectra of these objects, as a rule, contain large number of lines. It is important, then, to study the influence of multilevel energy spectrum of nanoparticles on their interaction. The present paper is devoted to modeling of laser induced dipole-dipole interaction of multilevel nanoparticles, for the cases when the particles are either identical or possess differing optical spectra.

\section{The model of nanoparticles' interaction in the laser radiation field}

The model of pair dipole-dipole interaction between nanoparticles is suitable for description of self-organization process. The particles involved into this process are ones that in the course of Brownian motion appeared to be at the distance where the interaction is large enough to influence their motion. Meanwhile, the energy of electrodynamic interaction of particles can exceed the thermal motion energy $k T$ and the energy of the barrier preventing uncontrolled aggregation. Therefore, laser-induced interparticle interaction can lead to the aggregation and formation of nanostructures with the configuration dictated by external field. [12-15]. We will also suppose that interparticle distance is much smaller than the wavelength of laser field, that allows to consider this filed as homogeneous, and retardation can be neglected.

With account for this, electric field vector of the light wave can be expressed as time-dependent harmonic function (c.c. in the formula (1))

$$
E=\frac{1}{2} E_{0}\left(e^{i \omega t}+e^{-i \omega t}\right) .
$$

Local field in the vicinity of every particle consists of external field and the field of induced dipoles. The dipole polarization at the frequency $\omega$ induced on $i$-th particle equals to

$$
\vec{d}_{i}=\chi_{0 i}\left(\vec{E}+\sum_{j} \vec{E}_{j}\right),
$$

where $\vec{E}_{j}$ is the strength of electric field produced by j-th particle in the point coinciding with the center of $i$-th particle, $\chi_{0 i}$ is the linear polarizability of isolated $i$-th particle, being equal to

$$
\chi_{0 i}=\sum_{k=1}^{K} \frac{|d|_{i k}^{2}}{\hbar\left(\Omega_{k}+i \Gamma_{i k}\right)},
$$


where $K$ is the number of allowed resonant transitions from the ground state to the $k$-th state, $\Omega_{k}=\left(\omega_{0 k}-\omega\right)$ is the detuning from $k$-th resonance, $w_{0 k}$ and $\omega$ are frequency of $k$-th transition of $i$-th particle and radiation frequency, $|d|_{i k}^{2}$ is the square of the modulus of electric dipole moment of $i$-th particle, $\hbar$ is the Planck constant, $\Gamma_{i k}$ is the homogeneous width of resonant line.

Assuming the intercenter distance between particles is much larger than particles' radii, we use point dipole approximation. Then electric field strength produced by $j$-th particle in the position of the center of $i$-th particle is described as

$$
\vec{E}_{j}=\frac{3\left(d_{j} r_{i j}\right) r_{i j}-d_{j} r_{i j}^{2}}{r_{i j}^{5}}
$$

where $r_{i j}=r_{i}-r_{j}$ is the intercenter distance between $i$-th and $j$-th particles.

Interaction energy of $i$-th and $j$-th laser-field-induced dipoles is

$$
W_{d i j}=\frac{d_{j} d_{j} r_{i j}^{2}-3\left(d_{i} r_{i j}\right)\left(d_{j} r_{i j}\right)}{r_{i j}^{5}}
$$

Let us consider the simplest case of interaction of a pair of nanoparticles in the field of linearly polarized laser radiation. The angle between polarization plane and intercenter direction is denoted as $\alpha$, and $x$ coordinate axis is chosen to be parallel to intercenter direction (Fig. 1).

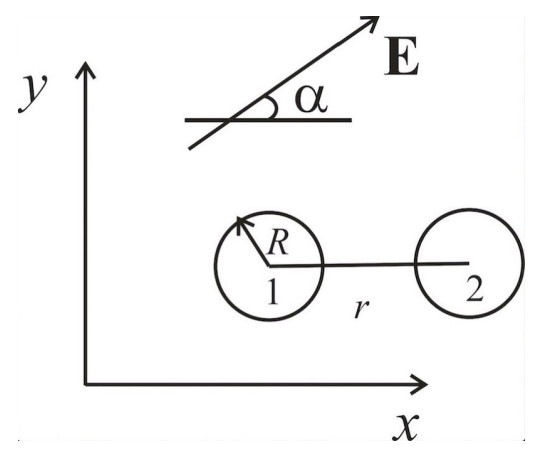

Fig. 1. Scheme of mutual orientation of the particles and the polarization of laser field

Energy of dipole-dipole interparticle interaction can be expressed through the amplitudes and phases of induced polarizations of every particle. Time-averaged expression (5) then will be

$$
W=\frac{\left|d_{1 y}\right|\left|d_{2 y}\right| \cos \left(\phi_{1 y}-\phi_{2 y}\right) \sin ^{2} \alpha-2\left|d_{1 x}\right|\left|d_{2 x}\right| \cos \left(\phi_{1 x}-\phi_{2 x}\right) \cos ^{2} \alpha}{2 r^{3}},
$$

where $\left|d_{1 y}\right|,\left|d_{2 y}\right|,\left|d_{1 x}\right|,\left|d_{2 x}\right|, \phi_{1 y}, \phi_{2 y}, \phi_{1 x}, \phi_{2 x}$ are modules and phases of $y$ and $x$ projections of time-averaged dipole polarization vectors of the first and second particles.

One can see from (6) that when polarization direction is parallel $(\alpha=0)$ or perpendicular $(\alpha=\pi / 2)$ to the intercenter direction, the sign of interaction energy is determined only by phase difference of corresponding dipole polarization projections. For identical particles, due to the index permutation symmetry, phases of induced polarizations projections coincide. For $\alpha=0$ interaction energy is negative, and this corresponds to attraction between particles, while for $\alpha=\pi / 2$ interaction energy is positive, and this leads to the repulsion of particles.

To be more specific, let us consider the interaction of colloidal QDs. Absorption lines of such solutions are inhomogeneously broadened due to polydispersity of particles, and quantities 
necessary for calculations using (3), (6) can hardly be extracted from experimental absorption spectra [22]. However, estimate for homogeneous linewidth $\Gamma$ and electric dipole moment of transitions $|d|_{i k}^{2}$ for solitary particle can be obtained using data on lumimescence of a single particle [22] and absolute values of absorption cross section [24]. For example, let us consider in the following the medium containing monodisperse CdSe particles with diameter 3,7 nm with the following parameters: homogeneous width of the first resonant peak $\Delta \lambda=3 \mathrm{~nm}$, square of the modulus of electric dipole moment $|d|_{i k}^{2}=1.91 \cdot 10^{-31} \mathrm{erg} \cdot \mathrm{cm}^{3}[22,25]$, width of inhomogeneously broadened first absorption peak $\Delta \lambda_{r}=30 \mathrm{~nm}$ [24]. Linear polarizability of isolated particle $\chi_{0 i}$ is worthwhile to present in the following form

$$
\chi_{0 i}=\sum_{k=1}^{K} \frac{|d|_{i k}^{2}}{\hbar\left(\Omega_{k}+i \Gamma_{i k}\right)}+\chi_{i n r}(\omega),
$$

where $K$ is the number of spectrally resolved absorption bands, $\chi_{i n r}(\omega)$ is the susceptibility of a single particle stipulated by spectrally unresolved transitions. Three spectrally resolved absorption bands were included in the calculation, namely, at 574, 543 and $478 \mathrm{~nm}$. Width of every band was $3 \mathrm{~nm}$, and relative values of electric dipole moments were estimated from their absorption cross sections [24]. Imaginary part of $\chi_{i n r}(\omega)$ was approximated with the account of the mean value of absorption cross section in the spectrally unresolved region by the expression

$$
\chi_{i n r}(\omega)=\frac{\left(\arctan \left(35 \omega-2.02 \cdot 10^{-17} c^{-1}\right)-\arctan \left(35 \omega-3.63 \cdot 10^{-17} c^{-1}\right)\right)}{\max \left(\arctan \left(35 \omega-2.02 \cdot 10^{-17} c^{-1}\right)-\arctan \left(35 \omega-3.63 \cdot 10^{-17} c^{-1}\right)\right)} \frac{|d|_{i k}^{2}}{\hbar i \Gamma_{i k}} \frac{\Delta \lambda}{\Delta \lambda_{r}} .
$$

For particles of unequal size we used resonant frequencies extracted from absorption spectra, while electric dipole moments and linewidths were taken to be equal to those of CdSe particles with $3,7 \mathrm{~nm}$ diameter.

In accordance with the approximations adopted above we recovered the imaginary part of linear susceptibility of CdSe particles with the radius 3,7 nm (Fig. 2a). Corresponding plot of real part of $\chi_{0 i}$ calculated via Kramers-Kronig relation [26] is plotted in Fig. $2 \mathrm{~b}$.
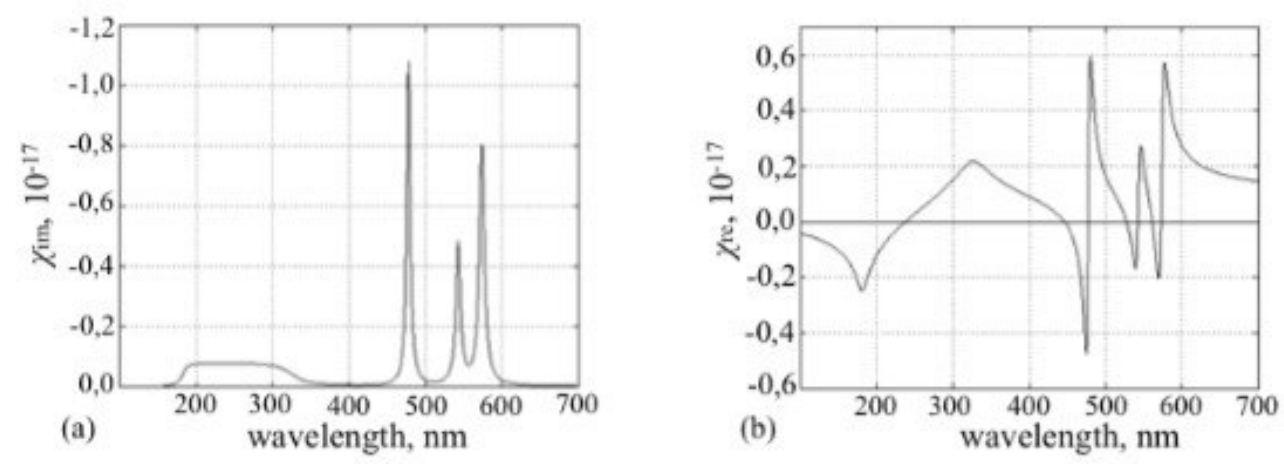

Fig. 2. Approximated imaginary (a) and real (b) parts of linear susceptibility of CdSe particle with the diameter $3,7 \mathrm{~nm}$

Approximations used make quantitative calculations of interaction energy to be of rather estimative character, however, results discussed below allow to achieve the qualitative understanding of the influence of multilevel nature of QDs' energy spectra on their electrodynamic interaction. 


\section{Results and discussion}

Calculations of interaction energy were done both for a pair of identical multilevel QD and for pair of QD with differing size. The latter case is more realistic due to polydispersity of colloidal QD solutions, and it also illustrates the possibility of structures' formation from QDs with differing resonant transition frequencies. Spectral dependence of interaction energy of a pair of particles at $\alpha=0$ normalized by $k T$ is plotted in Fig. 3. Solid line corresponds to interaction energy of two identical CdSe QDs with the diameter $3.7 \mathrm{~nm}$ and possessing linear polarizability depicted in Fig. 2. Dashed line corresponds to the spectral dependence of interaction energy of two different QDs with diameter 3.7 and $2.6 \mathrm{~nm}$. Linear polarizability of QD with $2.6 \mathrm{~nm}$ diameter was found similarly to that with $3.7 \mathrm{~nm}$, that is, from corresponding extinction spectrum. Resonant wavelengths of spectrally resolved transitions for QD with $2.6 \mathrm{~nm}$ diameter are 515 and $412 \mathrm{~nm}$. Parameters used in the calculations are the following: intercenter distance of QDs, temperature of the medium $300 \mathrm{~K}$, electric field strength $E=200 \mathrm{ESU}$, that corresponds to the intensity $10^{6} \mathrm{~W} / \mathrm{cm}^{2}$.
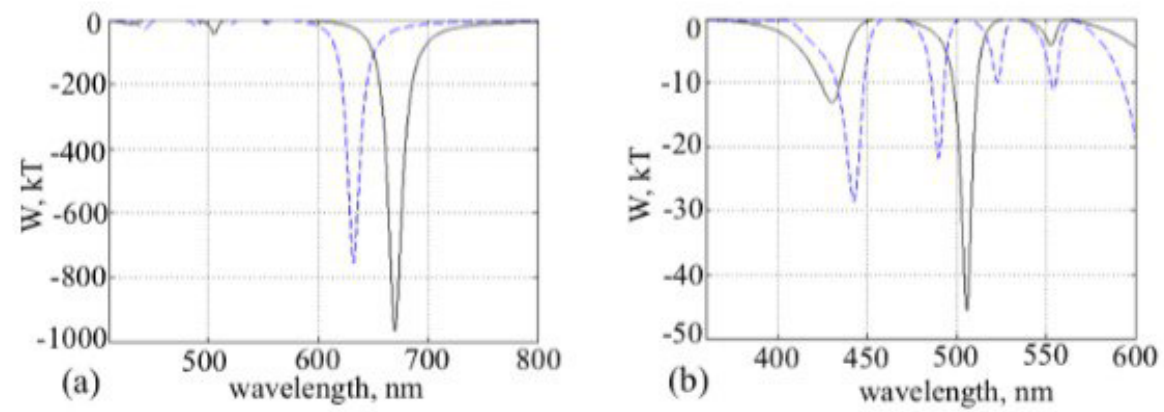

Fig. 3. (a) Interaction energy $W$ of two identical QDs with the diameter $3.7 \mathrm{~nm}$ (solid line) and two different QDs with diameters 3.7 and $2.6 \mathrm{~nm}$ (dashed line) as the function of external radiation wavelength. (b) Detailed plot of interaction energy in the range 350-600 nm

Data depicted in Fig. 3 show that the deepest potential wells are positioned in the region close to the most long wavelength transition of individual QD. This is understandable since detunings from resonances and, hence, contribution from all transitions to linear susceptibility (7) are of the same sign in this region. Resulting depth of potential well not only exceeds the thermal motion energy but is comparable to chemical bonds energy. Despite estimative character of our calculations, this result gives hope that the method under consideration is practically realizable. Fig. 3b illustrates that in shorter-wavelength region where contributions from "upper" and "lower" resonant bands are of opposite sign, interaction energy maxima are more than 20 times smaller than in the longest-wavelength potential well.

Spectral dependence of interaction energy for case of a pair of QDs with differing size and resonant frequencies is featured by smaller depth of potential wells. Influence of additional resonances still leads to deepening of potential wells as compared to the case of the model of two-level QD.

In a polydisperse QD solution, the QDs with different resonant wavelengths are present. Let us consider the interaction of a QD with a single resonance at $500 \mathrm{~nm}$ with another QD with arbitrarily positioned resonance. All the other characteristics used in the calculation of potential well parameters are taken for both QDs to be equal to those of CdSe QD with resonant wavelength 
$500 \mathrm{~nm}$. Dependences of the potential well depth (solid line) and position (dashed line) on the resonant wavelength of the second QD are plotted in Fig. 4. Vertical line corresponds to resonant

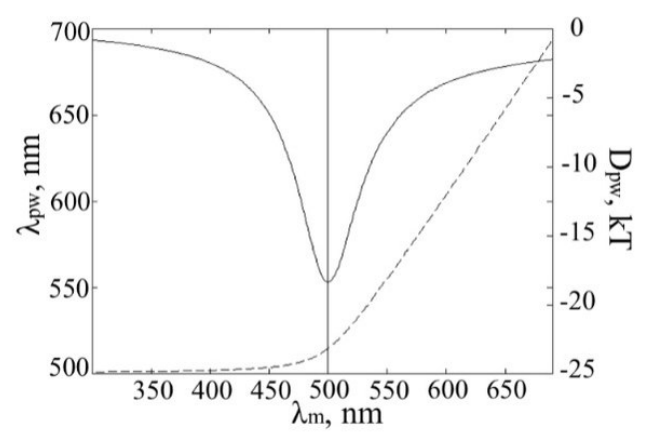

Fig. 4. Dependences of the potential well depth $D_{p w}$ (solid) and its spectral position $\lambda_{p w}$ (dashed) on the resonant wavelength of the second QD $\lambda_{m}$. Vertical line indicates the resonant wavelength of the first QD

wavelength of the first QD. The strongest attraction takes place for identical particles. Potential well depth dependence is asymmetric with the width at half maximum as wide as $75 \mathrm{~nm}$, the value being much larger than the absorption linewidth of individual particle $(3 \mathrm{~nm})$. Therefore, considerable decrease of interaction energy is expected at resonant wavelengths difference more than 25 times larger than QD linewidth. Position of the potential well, as can be seen from dashed curve in Fig. 4, is always shifted to the longer wavelength part of the spectrum with respect to the resonance of isolated particle with the most longer-wavelength individual resonance. At large difference of resonant frequencies this "red shift" is small and becomes maximum at the coincidence of frequencies.

Let us further estimate the influence of multilevel character of QD on the depth and position of potential wells. Let the first QD to possess two resonant transitions at wavelengths 400 and $500 \mathrm{~nm}$, while the second QD will have a single arbitrary positioned resonance. 3D dependence of interaction energy on the external field wavelength and the position of resonant transition of the second QD are plotted in Fig. 5. This dependence is featured by two deep potential wells

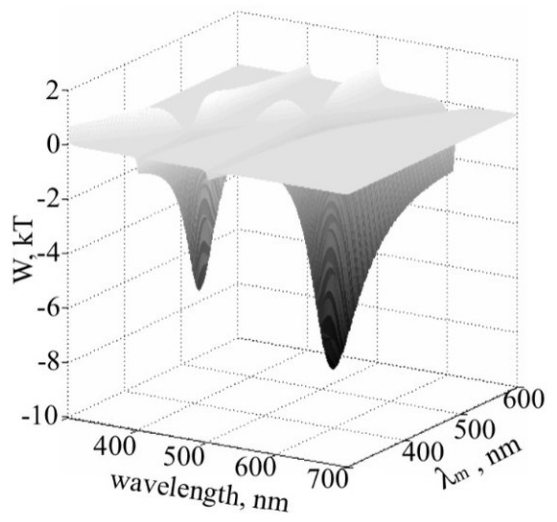

Fig. 5. 3D dependence of interaction energy on the external light wavelength and on the position of resonant transition of the second particle 
corresponding to resonant wavelengths of the first particle and by a rather complicated relief comprised by additional attracting minima and repulsive maxima. This relief must be associated with the dependence of phases of dipole polarizations induced at two transitions of the first QD and due to the interference of corresponding contributions to the interaction energy [14].

Fig. 6 depicts two most important potential wells of two-QD system with three resonances. Depths of potential wells are plotted by solid lines while dashed lines depict positions of potential wells at different position of the resonance of the second QD. These dependences are in general similar to those for single-resonance particles (Fig.4).
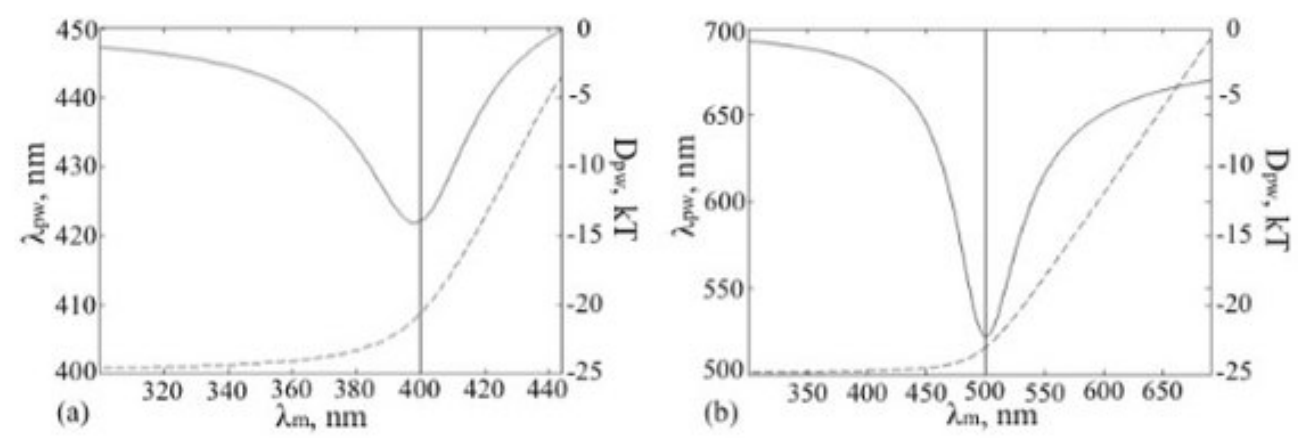

Fig. 6. Dependences of two potential wells' depths $D_{p w}$ (solid ) and its spectral position $\lambda_{p w}$ (dash) on the wavelength of the second QD transition $\lambda_{m}$. Vertical lines indicate resonant wavelengths of the first QD

\section{Conclusion}

Calculations of interaction energy of multilevel QD predict existence of attraction potential wells with the depths comparable with chemical bond energy at laser radiation intensity of order of $10^{6} \mathrm{~W} / \mathrm{cm}^{2}$.

The maximum potential well depth is expected for a laser wavelength in the vicinity of the most longer-wavelength resonance of the $\mathrm{QD}$, since contributions of all transitions in this region are of the same sign. In the ensemble of polydisperse QD, the strongest interaction takes place for a pair of identical particles. For a pair of non-identical QDs the dependence of potential well depth on the detuning is an asymmetric one in the wavelength scale. The spectral width of potential well can be up to $100 \mathrm{~nm}$, exceeding the absorption linewidth of individual QD more than by the order of magnitude. Maximum of attraction between QD is always red-shifted from the longest-wavelength resonance of both QDs, asymptotically approaching to it with the growth of resonance wavelengths' difference.

This research is partially supported by Grant of the Ministry of Education and Science of the Russian Federation for Siberian Federal University (projects 3.1749.2014/K, 214/71) and by RFBR, Government of Krasnoyarsk Territory, Krasnoyarsk Region Science and Technology Support Fund to the research project no. 16-42-240410. 


\section{References}

[1] J.Gong, G.Li, Z.Tang, Nano Today, 7(2012), 564.

[2] M.Rycenga, C.M.Cobley, J.Zeng, W.Li, C.H. Moran, Q.Zhang, D.Qin, Y.Xia, Chem. Rev, 111(2011), 3669 .

[3] S.Kinge, M.Crego-Calama, D.N.Reinhoudt, Chemphyschem, 9(2008), 20.

[4] T.Wang, D.LaMontagne, J.Lynch, J.Zhuang, Y.C.Cao, Chem. Soc. Rev, 42(2013), 2804.

[5] Z.Nie, A.Petukhova, E.Kumacheva, Nat. Nanotechnol., 5(2010), 15.

[6] M.Grzelczak, J.Vermant, E.M.Furst, L.M.Lizmarza, ACS Nano, 4(2010), 3591.

[7] M.Fialkowski, K.J.M. Bishop, R.Klajn, S.K.Smoukov, C.J.Campbell, B.A.Grzybowski, J. Phys. Chem. B, 110(2006), 2482.

[8] Y.Min, M.Akbulut, K.Kristiansen, Y. Golan, J.Israelachvil, Nat. Mater., 7(2008), 527.

[9] M.M.Mahlambi, A.K.Mishra, S.B.Mishra, A.M.Raichur, B.B.Mamba, R.W.Krause, Journal of Nanomaterials, Article ID 302046 (2012).

[10] P.Akcora, H.Liu, S.K.Kumar, J.Moll, Y.Li, B.C.Benicewicz, L.S.Schadler, D.Acehan, A.Z.Panagiotopoulos, V.Pryamitsyn, V.Ganesan, J.Ilavsky, P.Thiyagarajan, R.H.Colby, J.F.Douglas, Nature Materials, 8(2009), no. 4, 354-359.

[11] Kyung-Jong Noh, Hyo-Jin Oh, Bo-Ra Kim, Sang-Chul Jung, Wooseung Kang, Sun-Jae Kim, Journal of Nanomaterials, Article ID 475430 (2012).

[12] V.V.Slabko, G.G.Khachatryan, A.S.Aleksandrovsky, JETP Letters , 84(2006), 300.

[13] V.V.Slabko, A.S.Tsipotan, A.S.Aleksandrovsky, Photonics and Nanostructures - Fundamentals and Applications, 10(2012), no. 4, 636-643.

[14] V.V.Slabko, A.S.Tsipotan, A.S.Aleksandrovsky, Quantum Electron, 43(2013), no. 5, 458462.

[15] V.V.Slabko, A.S.Tsipotan, A.S.Aleksandrovsky, Appl. Phys. B, 117(2014), no. 1, 271-278.

[16] T.Hartling, Y.Alaverdyan, A.Hille, M.T.Wenzel, M.Kall, L.M.Eng, Optically controlled interparticle distance tuning and welding of single gold nanoparticle pairs by photochemical metal deposition, Optical Society of America, 16(2008), no. 16, 12362-12371.

[17] W.Rechberger, A.Hohenau, A.Leitner, J.R.Krenn, B.Lamprecht, F.R Aussenegg, Optical properties of two interacting gold nanoparticles, Optics Communications, 220(2003), no. $1-3,137-141$.

[18] N.Tate, M.Naruse, Y.Liu, T.Kawazoe, T.Yatsui, M.Ohtsu, Appl. Phys. B, 112(2013), $587-592$.

[19] V.Demergis, E.-L.Florin, Ultrastrong Optical Binding of Metallic Nanoparticles, Nano Lett., 12(2012), no. 11, 5756-5760. 
[20] F.Svedberg, Zhipeng Li, Hongxing Xu, M.Kall, Creating Hot Nanoparticle Pairs for SurfaceEnhanced Raman Spectroscopy through Optical Manipulation, Nano Lett, 6(2006), no. 12, 2639-2641.

[21] S.V.Karpov, A. L.Bas'ko, A.K.Popov, V.V.Slabko, Optics and Spectroscopy, 95(2003), no. 2, $241-247$.

[22] A.L.Rogach (Ed.), Semiconductor Nanocrystal Quantum Dots, Springer, Wien, NY, 2008.

[23] N.E.Lyamkina, G.A.Chiganova, V.V.Slabko, A.M.Vorotynov, M.A.Taranova, Inorganic Materials, 41(2005), no. 8, 830-835.

[24] C. de Mello Donega, R.Koole, J. Phys. Chem. C, 113(2009), no. 16, 6511-6520.

[25] M.Alves-Santos, R.Di Felice, G.Goldoni, J.Phys.Chem., 114(2010), 3776.

[26] P.Martin, Phys. Rev. B, 161(1967), 143.

\title{
Влияние многоуровнего характера спектра квантовых точек на энергию их взаимодействия в поле лазерного излучения
}

\author{
Андрей А. Глушков \\ Евгения А. Слюсарева \\ Александр С. Александровский \\ Алексей С. Ципотан \\ Виталий В. Слабко \\ Институт инженерной физики и радиоэлектроники \\ Сибирский федеральный университет \\ Свободный, 79, Красноярск, 660041 \\ Россия
}

$\overline{B \text { диполь-дипольном приближении произведен расчет энергетических спектров взаимодействия }}$ пары наночастии, CdSе в квазирезонансном лазерном поле для случаев двух одинаковых частии, и частии, с отличающимися длинами волн переходов. Показано, что учет дополнительных переходов значительно увеличивает величину энергии взаимодействия для потенциалъной ямы, лежащей в длинноволновой области спектра. На примере двух частии, с ограниченным числом резонансов исследовано влияние разности резонансных длин волн на глубину и положение потенциальной ямы. Показано, что глубина потенциальной ямы уменьшается при увеличении разности резонансных длин волн переходов двух частии.

Ключевые слова: диполь-дипольное взаимодействие, наночастичы и наноструктуры, взаимодействие лазерного излучения с веществом, дисперсионные соотношения. 